\title{
Identification of a novel polyfluorinated compound as a lead to inhibit human enzymes aldose reductase and AKR1B10: structure determination of both ternary complexes and implications for drug design
}

Alexandra Cousido-Siah, ${ }^{a,++}$ Francesc X. Ruiz, ${ }^{a, b,++}$ André Mitschler, ${ }^{a}$ Sergio Porté, ${ }^{b}$ Ángel R. de Lera, ${ }^{c}$ María J. Martín, ${ }^{d}$ Sonia Manzanaro, ${ }^{d}$ Jesús A. de la Fuente, ${ }^{d}$ Felix Terwesten, ${ }^{e}$ Michael Betz, ${ }^{e}$ Gerhard Klebe, ${ }^{\text {e }}$ Jaume Farrés, ${ }^{\text {b }}$ Xavier Parés, ${ }^{b}$ Alberto Podjarny ${ }^{\mathbf{a}^{*}}$

aDepartment of Integrative Biology, Institut de Génétique et de Biologie Moléculaire et Cellulaire, 1 rue Laurent Fries 67404 Illkirch CEDEX, France

${ }^{b}$ Department of Biochemistry and Molecular Biology, Universitat Autònoma de Barcelona, E-08193

Bellaterra, Barcelona, Spain

`Departamento de Química Orgánica, Universidade de Vigo, E-36310 Vigo, Spain

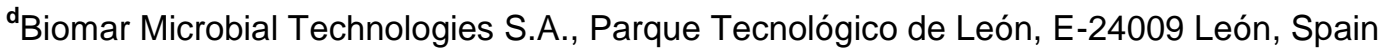

eDepartment of Pharmaceutical Chemistry, University of Marburg, Marbacher Weg 6, D-35032

Marburg, Germany

${ }^{++}$These authors contributed equally to this work

"To whom correspondence should be addressed: e-mail: podjarny@igbmc.fr

PDB references:

Crystal structure of human Aldose Reductase complexed with NADP ${ }^{+}$and JF0064, 4IGS

Crystal structure of human AKR1B10 complexed with NADP ${ }^{+}$and JF0064, 4ICC 


\section{Supporting Information}

\section{S1. Material and methods}

\section{S1.1. Molecular dynamics}

The molecular dynamics (MD) simulations were performed using AMBER 12 and the SPFP precision model (Le Grand et al., 2013), analysis of the trajectory was carried out using PTRAJ and CPPTRAJ (Roe \& Cheatham, 2013) as implemented in Amber Tools 12 (Case et al., 2012; Goetz et al., 2012; Salomon-Ferrer et al., 2013). Molecular preparation of the protein-JF0064 complexes was carried out using MOE (version 2012.10, Chemical Computing Group, Montreal, Canada, http://www.chemcomp.com). The input structure of protein and ligand was prepared from the here reported mAKR1B10 NADP ${ }^{+}: K 125 R / V 301 L: J F 0064$; for the wild type structure residues Arg125 and Leu301 were mutated to Lys125 and Val301 in silico using MOE.

Protonation of the AKR1B10:JF0064 complexes was carried out using the Protonate3D function of MOE by applying the default values except the dielectric value that was set to 80 . For both proteinligand complexes, Asp and Glu residues were negatively charged and all Lys and Arg residues were found to be positively charged. Four doubly protonated His were found (residues 42, 164, 201, 241) and five were protonated on NE2 of His (residues 53, 111, 188, 253, 306). Then the ligand and the cofactor $\mathrm{NADP}^{+}$were charged separately using the AM1-BCC charge model (Jakalian et al., 2000; Jakalian et al., 2002).

The preprocessed proteins, ligands and cofactors were parameterized with the program antechamber using the generalized AMBER force field (GAFF) (Wang et al., 2004) for the small organic molecules and the ff99SB force field (Hornak et al., 2006) for the proteins. Electroneutrality of the system was maintained by adding chloride or sodium ions as counterions followed by placing each starting structure in a truncated octahedral periodic box of TIP3P water molecules (Jorgensen et al., 1983). The closest distance between the edges of the water box and any distance of the solute was set to $10 \AA$. MD simulations were performed with pmemd.cuda on Nvidia K20 graphic cards under periodic boundary conditions using the particle mesh Ewald method (Darden et al., 1993) with a cut-off of $10 \AA$ A. The SHAKE algorithm (Ryckaert et al., 1977) was applied to constrain bonds involving hydrogen. Langevin dynamics (Loncharich et al., 1992) with a predefined collision frequency of $5 \mathrm{ps}^{-1}$ between external heat reservoir and simulated system were used to adjust temperature and isotropic position scaling to regulate pressure. The time steps of the simulation were set to $2 \mathrm{fs}$.

The water box was minimized by 100 cycles of steepest descent minimization followed by 400 cycles of conjugate gradient minimization. Afterward, the system consisting of water and protein-ligand complex was minimized by 200 cycles of steepest descent minimization followed by 1000 cycles of 
conjugate gradient minimization. The system was gradually heated from 0 to $300 \mathrm{~K}$ in $150 \mathrm{ps}$ using Cartesian restraints of $500 \mathrm{kcal} \mathrm{mol}^{-1} \AA^{-2}$ on the complex followed by adjusting the pressure in $100 \mathrm{ps}$ to 1 bar. Finally, before running the MD simulation, the restraints on the complex were set gradually to 0 in $400 \mathrm{ps}$. The 110-ns MD simulations were performed storing the coordinates along the trajectory every $10 \mathrm{ps}$.

For AKR1B10 and AKR1B10 K125R/V301L, each in complex with JF0064, several 110-ns MD simulations were carried out (for the ligand with protonated hydroxyl groups and the ligand containing the deprotonated hydroxyl group at position 4).

Distances were calculated for individual ligand atom-protein atom pairs using the distance command implemented in PTRAJ. For the second 110-ns MD simulation of the AKR1B10:NADP ${ }^{+}$:JF0064 complex (unprotonated ligand) a clustering of the ligand and the amino acids participating in binding was carried out (Tyr49, His111, Trp112, Phe123, Trp220, Val301, Gln303). As distance metric the RMSD was used, for calculating the distances between the clusters the average distance was applied. The program was constrained to find 25 clusters. In Figure 6 the first cluster populated with 5849 frames (53.2\% of this productive MD) is shown. For this cluster water molecules were added following the above described method, deviating by using Cartesian restraints of $500 \mathrm{kcal} \mathrm{mol}^{-1} \AA^{-2}$ on the complex for the entire simulation, and finishing the simulation after heating with a $1 \mathrm{~ns}$ productive MD simulation and an additional minimization. The RMSD between the ligand of the input file and the ligand in every frame of the MD (see RMSD files) was calculated using VMD (v1.9.1) (Humphrey et al., 1996).

\section{S1.2. Chemicals}

The series of JF inhibitors were provided by Biomar Microbial Technologies S.A. and synthesized as described previously. JF0025, JF0042, JF0053 and JF0246 were compounds 15, 24, 27 and 13, respectively, in de la Fuente and coworkers (de la Fuente et al., 2003). JF0127 was compound 3 in Jarman and coworkers (Jarman et al., 1990). JF0064 was commercially obtained from Sigma-Aldrich. Synthesis of JF0021 was described by Martin (Martin, 2000). Synthesis of 3,3',5,5'-tetrabromo2,2',4,4'-tetrahydroxybenzophenone (JF0143): To a solution of 2,2',4,4'-tetrahydroxybenzophenone (200 mg, $0.81 \mathrm{mmol})$ in acetic acid $(5 \mathrm{~mL})$, at room temperature, $0.5 \mathrm{~mL}(9.72 \mathrm{mmol})$ bromine were added by stirring. The mixture was heated for $5 \mathrm{~h}$ under reflux, then cooled to room temperature and concentrated in vacuo. The residue was dissolved in $\mathrm{CCl}_{4}: \mathrm{CHCl}_{3} \quad 1: 1$ and crystallized from this mixture to yield JF0143 as an orange solid (324 mg, 71\%): mp: $262-264^{\circ} \mathrm{C} ;{ }^{1} \mathrm{H}$ NMR $\left(\mathrm{CD}_{3} \mathrm{OD}\right): \delta 7.54$ $(\mathrm{s}, 2 \mathrm{H}) ;{ }^{13} \mathrm{C}$ NMR $\left(\mathrm{CD}_{3} \mathrm{OD}\right): \delta 197.92,158.53,157.82,135.40,117.53,101.48,101.31$; MS (APCInegative) $\mathrm{m} / z: 561(100 \%)[\mathrm{M}-\mathrm{H}]^{-}, 481[\mathrm{M}-\mathrm{Br}]^{-}$. The melting point was determined in open capillaries with a Büchi B-535 melting point apparatus and is uncorrected. The NMR spectra were recorded on a 
Varian 300 spectrometer at $300 \mathrm{MHz}$ for ${ }^{1} \mathrm{H}$ NMR and at $75 \mathrm{MHz}$ for ${ }^{13} \mathrm{C} \mathrm{NMR}$, with tetramethylsilane as an internal standard. Chemical shifts $(\delta)$ are reported as s (singlet). Atmospheric Pressure Chemical Ionization (APCI) mass spectra were recorded on a HP 1100 LC/MS. All reagents were used as received unless otherwise stated.

\section{S2. Results and discussion}

\section{S2.1. Medicinal chemistry analysis of the series of polyfluorinated compounds}

Regarding $\mathrm{AR} \mathrm{IC}_{50}$ assays, the most potent inhibitor was clearly JF0064 $\left(\mathrm{IC}_{50}=0.3 \mu \mathrm{M}\right)$, with a selectivity of 3.4-fold for AR versus AKR1B10. The remaining part of assayed compounds showed $\mathrm{IC}_{50}$ values ranging from 3.2 to $8.9 \mu \mathrm{M}$. JF0021 and JF0042 presented a selectivity ratio of more than 6-fold for AR, while the other inhibitors had similar values for the two enzymes. JF0246 exhibited a high $\mathrm{IC}_{50}$ value, above $46 \mu \mathrm{M}$, as reported previously, and was used as a negative control of the series (de la Fuente et al., 2003) (Table 1).

With respect to AKR1B10 $\mathrm{IC}_{50}$ assays, several compounds showed low micromolar values: JF0064, with $1.0 \mu \mathrm{M}$ being the most potent, while JF0025, JF0053, JF0127 and JF0143 exhibited values between 1.6 and $3.4 \mu \mathrm{M}$. The other analogues showed values higher than $20 \mu \mathrm{M}$. JF0143, JF0025, and JF0127 were the most selective compounds for AKR1B10, between 2 and 4-fold (Table 1).

Marine natural products were the starting point for the synthesis of a series of polyhalogenated derivatives ARIs (de la Fuente et al., 2003) (Figure 1B). Indeed, marine animals have demonstrated to be rich sources of halogenated metabolites with promising drug inhibition properties (de la Fuente \& Manzanaro, 2003; de la Fuente et al., 2003; Fenical et al., 2009; Manzanaro et al., 2006; Shinde et al., 2008). In this work, those derivatives having $\mathrm{IC}_{50}$ values below $10 \mu \mathrm{M}$ with respect to $\mathrm{AR}$ inhibition were also tested against AKR1B10. They can be divided into three groups. The first group is composed of the benzophenone analogues JF0021 and JF0143. Both of them are poor AR inhibitors, but their potency against AKR1B10 differs approximately by 18 -fold (Table 1). The cause seems to be the introduction in JF0143 of two hydroxyl groups in $\mathrm{R}_{3}$ and $\mathrm{R}_{8}$ (instead of $\mathrm{H}$ atoms in JF0021, Figure 1B).

The second group is formed by JF0025, JF0042, JF0053 and JF0246. JF0025 and JF0053 are very similar in structure and in inhibition properties for both enzymes. The loss of inhibition by JF0246, with sterically bulkier methyl ethers, suggests that the hydroxyl groups at $R_{1}$ and $R_{6}$ positions of JF0025 might play a significant role in enzyme binding (as in compound 3, Figure 1B,Table 1) via the direct involvement of one or both hydroxyl groups in significant interactions with the enzyme binding site (de la Fuente et al., 2003). This is also supported by the decrease of inhibitory potency of JF0042 
against both enzymes (especially for AKR1B10), with the only difference of an amino instead of a hydroxyl group at $\mathrm{R}_{6}($ Table 1$)$.

The third group is formed by JF0064 and JF0127. The former compound exhibited the lowest $\mathrm{IC}_{50}$ values for each of the two enzymes, with a scaffold (1,1'-biphenyl-4,4'-diol) that differs from the carboxylic acid or cyclic imide moiety (Manzanaro et al., 2006). JF0127 is similar to JF0064, but with an azo group inserted between the tetrafluorophenol groups (Figure 1B). JF0127 is a much less potent inhibitor against AR, while keeping the AKR1B10 inhibitory potency (Table 1), as the tetrafluorophenol moieties of JF0127 are likely to display different relative orientations due to the presence of the azo group.

\section{S2.3. Molecular dynamics}

Rough assessment of the ligand conformation stability was done using calculated distances between several ligand atom-protein atom pairs and the RMSD between the ligand of the input file and the ligand in every frame of the MD. The labels of the distances are shown in S1A and S1B the corresponding plots are provided in the separate files: AKR1B10_distances.pdf and AKR1B10K125R.V301L_distances.pdf. The fluctuation of the RMSDs is shown in the RMSD files, accordingly. For comparison with the crystal structures only the parts of the MD were analysed in which the ligand was fixed in the binding pocket. This information can be easily extracted from the RMSD and distance plots. Distances A and B in the plots show almost perfect hydrogen bond distances. Additionally, the values for the RMSD of the ligand are also very low. In both systems, AKR1B10:JF0064 and AKR1B10 K125R/V301L:JF0064, there are two main conformations of the ligand preferentially populated in the MD simulations. Firstly, the one in which the ligand is fixed in the pocket, and secondly, the binding pose in which the ligand forms a hydrogen bond with Lys/Arg125. In the distance plots this is shown in distance C. Simultaneously, distance A displays the ligand losing the short contact between His111 and its inner hydroxyl group. 


\section{Figure S1}

A

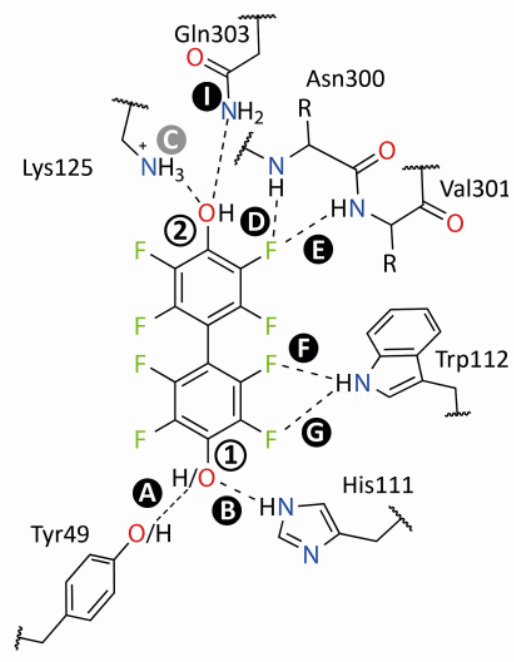

B

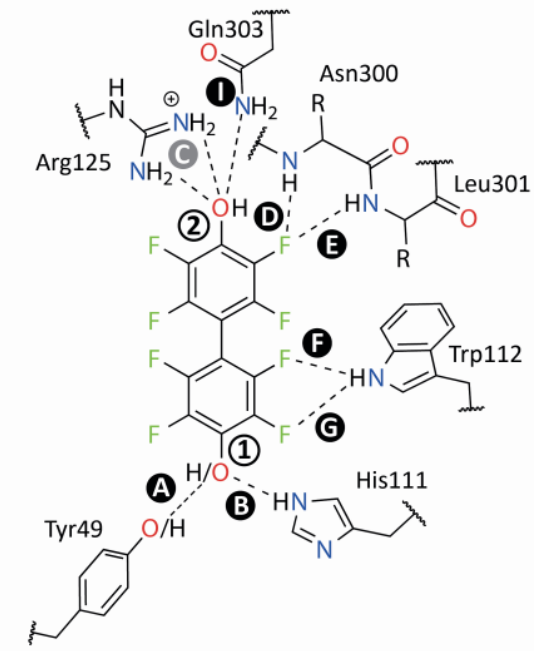

Supporting Figure S1. Representation of the binding pockets of the complexes simulated by molecular dynamics simulations. (1) depicts the hydroxyl group of the inner tetrafluorophenol moiety and (2) the hydroxyl group of the distal one. Labels (A) to (I) represent the calculated distances plotted in the distances files. Distance (A) represents the hydrogen bond established between the oxygen of Tyr49 and the hydrogen of the hydroxyl group (for the protonated ligand), and the hydrogen of Tyr49 and the oxygen of the hydroxyl group of the ligand (for the deprotonated ligand). Distance (C) is only observable if the ligand leaves its native binding mode. Figure S1A shows the distances for the AKR1B10:JF0064 complex, and S1B for AKR1B10 K125R V301L:JF0064 accordingly. Note that both possible protonation states of the proximal hydroxyl group have been simulated. 


\section{Supporting References}

Case, D. A., Darden, T. A., Cheatham III, T. E., Simmerling, C. L., Wang, J., Duke, R. E., Luo, R., Walker, R. C., Zhang, W., Merz, K. M., Roberts, B., Hayik, S., Roitberg, A., Seabra, G., Swails, J., Goetz, A. W., Kolossváry, I., Wong, K. F., Paesani, F., Vanicek, J., Wolf, R. M., Liu, J., Wu, X., Brozell, S. R., Steinbrecher, T., Gohlke, H., Cai, Q., Ye, X., Wang, J., Hsieh, M. J., Cui, G., Roe, D. R., Mathews, D. H., Seetin, M. G., Salomon-Ferrer, R., Sagui, C., Babin, V., Luchko, T., Gusarov, S., Kovalenko, A. \& Kollman, P. A. (2012). AMBER 12, University of California, San Francisco, http://ambermd.org.

Darden, T., York, D. \& Pedersen, L. (1993). Journal of Chemical Physics 98, 10089-10092.

de la Fuente, J. A. \& Manzanaro, S. (2003). Nat Prod Rep 20, 243-251.

de la Fuente, J. A., Manzanaro, S., Martín, M. J., de Quesada, T. G., Reymundo, I., Luengo, S. M. \& Gago, F. (2003). J Med Chem 46, 5208-5221.

Fenical, W., Jensen, P. R., Palladino, M. A., Lam, K. S., Lloyd, G. K. \& Potts, B. C. (2009). Bioorg Med Chem 17, 2175-2180.

Goetz, A. W., Williamson, M. J., Xu, D., Poole, D., Le Grand, S. \& Walker, R. C. (2012). Journal of Chemical Theory and Computation 8, 1542-1555.

Hornak, V., Abel, R., Okur, A., Strockbine, B., Roitberg, A. \& Simmerling, C. (2006). ProteinsStructure Function and Bioinformatics 65, 712-725.

Humphrey, W., Dalke, A. \& Schulten, K. (1996). J Mol Graph 14, 33-38, 27-38.

Jakalian, A., Bush, B. L., Jack, D. B. \& Bayly, C. I. (2000). Journal of Computational Chemistry 21, 132-146.

Jakalian, A., Jack, D. B. \& Bayly, C. I. (2002). Journal of Computational Chemistry 23, 1623-1641.

Jarman, M., Barrie, S. E., Deadman, J. J., Houghton, J., McCague, R. \& Rowlands, M. G. (1990). J Med Chem 33, 2452-2455.

Jorgensen, W. L., Chandrasekhar, J., Madura, J. D., Impey, R. W. \& Klein, M. L. (1983). Journal of Chemical Physics 79, 926-935.

Le Grand, S., Goetz, A. W. \& Walker, R. C. (2013). Computer Physics Communications 184, 374380.

Loncharich, R. J., Brooks, B. R. \& Pastor, R. W. (1992). Biopolymers 32, 523-535.

Manzanaro, S., Salva, J. \& de la Fuente, J. A. (2006). J Nat Prod 69, 1485-1487.

Martin, R. (2000). Handbook of hydroxybenzophenones, p. 423. Dordrecht (The Netherlands): Kluwer Academic Publishers.

Roe, D. R. \& Cheatham, T. E. (2013). Journal of Chemical Theory and Computation 9, 3084-3095.

Ryckaert, J.-P., Ciccotti, G. \& Berendsen, H. J. C. (1977). Journal of Computational Physics 23, 327341.

Salomon-Ferrer, R., Götz, A. W., Poole, D., Le Grand, S. \& Walker, R. C. (2013). Journal of Chemical Theory and Computation 9, 3878-3888.

Shinde, P. B., Lee, Y. M., Dang, H. T., Hong, J., Lee, C. O. \& Jung, J. H. (2008). Bioorg Med Chem Lett 18, 6414-6418.

Wang, J. M., Wolf, R. M., Caldwell, J. W., Kollman, P. A. \& Case, D. A. (2004). Journal of Computational Chemistry 25, 1157-1174. 